\title{
Congregations as Health Connectors - Addressing Health and Social Needs of Neighbors Through Conversations, Not Surveys
}

\author{
Kristen Buss ${ }^{1}$, David Craig $2 ; 3$, Emily Hardwick ${ }^{3}$, Sarah Wiehe ${ }^{3}$ \\ ${ }^{1}$ Indiana University School of Medicine, ${ }^{2}$ IUPUI, Department of Religious Studies, ${ }^{3}$ Indiana \\ Clinical and Translational Sciences Institute, Monon Collaborative
}

Background and Hypothesis: Social determinants of health (SDOH) directly affect health outcomes and indirectly limit access to resources needed to maintain individual health. In an effort to address the negative impacts of SDOH on the urban communities of Indianapolis, four congregations have employed Site Connectors to directly form relationships with neighbors, learn about their health journey, and connect them to resources addressing expressed needs. It is our hypothesis that through research of similar models and discussion with community partners, an optimized model for fostering relationships and assessing the health and social needs of neighbors can be developed for use by the Site Connectors.

Project Methods: A search was performed for examples of health and social needs assessments utilized by established care providers, and thirteen were identified. The items within these assessments were then organized into six groups based on $\mathrm{SDOH}$ topic (ie. Housing, Transportation, etc.). Additionally, six interviews with community partners performing similar work were conducted utilizing a fixed set of questions.

Results: It was determined that the best format for our assessment would not be a survey, as in the example assessments, but rather a visual aid resembling a concept map. This model lends itself more to the nature of relationship-building by guiding Connector-Neighbor conversations rather than dictating them, with three starter questions at the center and six offshoots covering each of the SDOH topics. A post-encounter checklist was also developed for Connectors to retroactively record priority items from their conversations.

Potential Impact: It is yet to be determined whether our model will be useful in practice, as the Connectors begin their work in August. However, it is our hope that we have developed a novel format for assessing needs that more holistically addresses the impacts of SDOH through respecting the vulnerability and energy required for relationship-building. 